\title{
Vom ungeliebten Kind zum geschätzten Familienmitglied?
}

\author{
Eine Nachbetrachtung
}

Anton Fischer (Zürich)

Was haben die fünf Vorträge des Winterzyklus gebracht, die sich der Leser in diesem Heft in überarbeiteter Form zu Gemüte führen kann? Ihre Absicht war es, das Verhältnis zwischen Psychoanalyse und Psychotherapie zu klären oder wenigstens einen Beitrag dazu zu leisten. Eine Klärung, die für unser Seminar nicht bloss von theoretischem, sondern auch von einem eminent praktischen Interesse ist: Es geht darum, sie nicht länger gegen einander auszuspielen, wie es oft genug geschah, sondern sie mehr oder weniger friedlich unter einem Dach koexistieren zu lassen und um denkbare Synergien. Oder, in den Worten von Thomas Merki: Das eine tun und das Andere nicht lassen.

Seit das PSZ in den Neunzigerjahren den Grundsatzentscheid gefällt hat, eine Weiterbildung in psychoanalytischer Psychotherapie anzubieten, hat es sich radikal verändert. Dies gefällt Vielen nicht. Wir können unsere hoch besetzten essentials wie Laienanalyse, Selbstautorisation oder Ablehnung der Lehranalyse nur noch für die Ausbildung zum Psychoanalytiker hochhalten und sind im anderen Bereich teilweise zum Vollzugsorgan fremder Erlasse geworden. Wir haben inzwischen zwei kulturell total verschiedene Regelsysteme im Hause PSZ, das frühere selbst bestimmte für die analytische Aus- und das neue fremdbestimmte für die therapeutische Weiterbildung. Das kann gar nicht ohne Friktionen abgehen. Umso mehr als es fraglich ist, ob in Zukunft Absolventen einer psychoanalytischen Ausbildung, die nicht zugleich Psychotherapeuten sind (und die gesetzlichen Anforderungen erfüllen) als reine Psychoanalytiker überleben können.

Haben wir damit unsere Seele verkauft, wie kritische Stimmen es befürchten? Oder hat der damalige Grundsatzentscheid uns vor einem sonst unvermeidbaren Bedeutungsverlust gerettet? So oder so ist nicht zu übersehen, wie viel Kraft und Kreativität die Auseinandersetzungen um die Modalitäten der psychotherapeutischen Weiterbildung verbraucht, allein schon die simple Frage, was sie kosten soll, blockiert die Teilnehmerversammlung stundenlang. In den Hintergrund gedrängt wurde dadurch die wichtigste Frage, nämlich die nach der Zukunft des PSZ. Obwohl die Gründergeneration langsam abtritt und damit die von ihr getrage- 
nen Ideale - basisdemokratische Selbstverwaltung, Selbstbestimmung, Solidarität zwischen Lehrenden und Lernenden - ihre bisherige Selbstverständlichkeit verlieren. Umso mehr als die Autonomie, die wir seinerzeit der in der IPA organisierten Psychoanalyse abgetrotzt haben, ohnehin nur noch für einen Teil des PSZ gilt. So haben wir den Widerspruch mitten unter uns. Wenn der letzte Achtundsechziger ins Altersheim gezogen ist, stellt sich die Selbstrechtfertigung der Existenz des PSZ oder-neudeutsch - die Frage nach der unique selling position neu. Weshalb braucht es uns noch? Was unterscheidet uns von den beiden anderen psychoanalytischen Ausbildungsstätten in Zürich? Was haben wir zu bieten, um die Wettbewerbsvorteile der Anderen wettzumachen? Wir können weder den Familienanschluss an die grosse IPA-Familie bieten (wie das Freud-Institut), noch akademische Titel verleihen (wie Frau Boothe an der Uni).

Martin Kuster weist auf die schnöde Realität in den psychoanalytischen Praxen hin. Gemäss der Umfrage der IPA unter ihren Mitgliedern von 1999 arbeiten fast alle Psychoanalytiker auf der ganzen Welt längst mehrheitlich als Psychotherapeuten. Mit der traditionellen Analyse allein können sie ihren Lebensunterhalt nicht mehr bestreiten. Das gelingt nur noch den Lehranalytikern, die wir ja in Zürich nicht wollen. Unter den PSZ-Analytikern sieht es nicht anders aus. Eine rein psychoanalytische Praxis unterhält auch da kaum jemand mehr (Ausnahmen melden sich bitte bei der Redaktion). Daher erweisen sich Debatten wie: Dürfen sich Psychoanalytiker an der Ausbildung von Nicht-Analytikern zu Psychotherapeuten beteiligen, als völlig absurd. Noch absurder ist, wie Kuster eindrücklich belegt, mit welcher Herablassung Vertreter der offiziellen Psychoanalyse die nämliche Psychotherapie behandelt haben, von der sie praktisch lebten. Die Folge davon war, dass sie während dreier Jahrzehnte buchstäblich keinen einzigen neuen Beitrag zum psychoanalytischen Verständnis von Psychotherapie lieferten. Sie wurde als das definiert, was sie nicht ist und praktisch als das, was zu tun übrig bleibt, wenn die Patienten sich als unfähig erweisen, die Entbehrungen der Psychoanalyse auf sich zu nehmen. Und das waren derer viele in der Epoche, als die Standard Technik in Blüte stand, mit einem Analytiker, der nichts sagt oder fragt, sondern allein gelegentlich eine Deutung von sich gibt. Wesentlich mehr als die Profilneurose von puristischen Psychoanalytikern drückt sich darin nicht aus. Sie erhielt ohnehin durch die Menningerstudie einen zweiten Schlag, die zeigte, dass in Psychoanalysen nicht allein die Deutungen wirksam sind.

Aus dieser peinlichen Geschichte bleibt die Frage zurück, ob eine sinnvolle Definition von Psychotherapie überhaupt von einer analytischen Position aus formuliert werden könne, oder ob es nicht vielmehr ein tertium comparationis 
bräuchte. Praktisch ergibt sich daraus die Einladung, endlich die Kränkungskröte zu schlucken, dass (zumindest in der Gegenwart) die Nachfrage nach Psychoanalysen nicht ausreicht, um einen Mann oder eine Frau standesgemäss zu ernähren. Ob es früher wesentlich anders war, das wissen wir nicht genau. Zweifelhafter Vorteil ist, dass wir uns nicht mehr fragen müssen, ob wir das reine Gold der Psychoanalyse mit minderwertigem Metall legieren wollen oder nicht. Diesen Entscheid nimmt uns der Markt ab.

Passett weist nach, wie viel gemeinsames Erbgut Psychoanalyse und Psychotherapie miteinander teilen, ähnlich viel nämlich wie Mensch und Schimpanse, und kritisiert den falschen Gegensatz, der häufig zwischen beiden konstruiert werde. Er polemisiert mit gewohnter Verve gegen die Vorstellung von der «reinen» Psychoanalyse und der verdünnten Psychotherapie, die so zur Schmalspuranalyse degradiert werde. Wer behauptet, dass das Phänomen der Übertragung überall vorkomme, kann nicht zugleich so tun, als ob die beiden Welten auseinander klafften. Echt psychoanalytische Momente gebe es auch in den vermeintlich reinen Analysen höchst selten, sie ragten wie Gipfelerlebnisse sozusagen aus dem Ozean alltäglicher psychotherapeutischer Knochenarbeit empor, die genau so stützend und suggestiv operiere wie in den angeblich kupferlegierten Psychotherapien. Aber um ihretwillen lohne sich das etwas altmodische Unterfangen, das durch «keinen materiellen Gewinn und keine Mehrheitsbedürfnisse garantiert» sei und man möge deshalb die Psychoanalyse als eine Art von gefährdetem Kulturgut schützen wie die Lyrik und die Schnabeltiere. Anwesende Funktionäre, die um die Kassenzulassung kämpfen, werden sich die Haare gerauft haben.

Was Passett an der Gegenüberstellung zweier Fallgeschichten erläutert, habe ich selbst über eine historische Herleitung zu begründen versucht. Ich verstehe das Interesse der Psychoanalyse für die Psychotherapie aus dem gesellschaftlichen Kontext, in dem es ursprünglich entstanden ist: die sozialdemokratische Gesundheitspolitik des Roten Wien. Freud war das neurotische Massenelend nicht gleichgültig, er fragte sich, wie es angegangen werden könnte - angesichts der Tatsache der geringen Reichweite der klassischen Analyse. Zweitens wollte ich zeigen, warum dieses Interesse ebenso rasch, wie es entstanden ist, wieder verschwunden ist, nämlich indem der Faschismus die Arbeiterbewegung total vernichtet hat, und die deutschsprachigen Analytiker in den angelsächsischen Raum geflüchtet sind. Drittens, dass ihnen die Psychotherapie nach dem Untergang des Faschismus mit Notwendigkeit als etwas von aussen Kommendes und Artfremdes entgegentrat, das sie nicht mehr als ihr eigenes Kind wieder erkannten. 
Nicht ganz überraschend plädiert der Präsident, inzwischen Expräsident des Schweizer Psychotherapeutenverbandes, Thomas Merki wie damals sein Vorgänger Freud für eine selbstbewusste Integration der Psychoanalyse in die heute real existierende seelische Gesundheitsversorgung. Er vertraut sozusagen auf ihren therapeutischen Mehrwert, der darin bestehe, dass sie keine schnelle Symptomheilung, sondern Veränderungen in der Persönlichkeitsstruktur bewirkt, die eine nachhaltigere (und damit letztlich effektivere) Wirkung erziele als vermeintlich kostengünstigere Eilverfahren. Er ist auch überzeugt, dass ihre Arbeit mit dem Unbewussten und der Übertragung, die ihr Markenzeichen darstellt, immer wieder die nötige wissenschaftliche Anerkennung findet und die Gesundheitspolitik sie nicht ignorieren könne.

RonyWeissberg untersucht in seinem Schlussvortrag Differenz und Gemeinsamkeit zwischen Therapie und Analyse, indem er sozusagen die Rohmaterialien isoliert, mit denen beide arbeiten. Er weist nach, eine wie wichtige Rolle in beiden Leidenschaft, aber auch die Liebe spielt - und überraschenderweise auch der Glaube, den er anstelle des Begriffs «Suggestion» setzen möchte. Freud spricht nämlich von der Übertragungsliebe, die den Glauben des Analysanden an den Therapeuten befördere und ihm dadurch überhaupt erst ermögliche, die Einsichten zu assimilieren, mit der ihn dieser konfrontiert. Die Fetischisierung von Abstinenz, Neutralität und Deutung seien nichts Anderes als eine zur Ideologie geronnene Abwehr der (eigenen) Leidenschaft (Lacans «Begehren») und den bedrohlich empfundenen Triebkräften des Analysanden, denen der Analytiker ausgesetzt ist. Weissberg beleuchtet Freuds merkwürdige Unentschiedenheit gegenüber der Psychotherapie, die einerseits von der «richtigen», strengen, tendenzlosen Analyse die Abstinenz übernehmen soll, also dieWeigerung irgendwelcheWünsche des Patienten zu befriedigen, aber auf der anderen Seite die Aktivität des zum Therapeuten werdenden Analytikers postuliert.

Wie Passett richtig feststellt, hat die Metapher vom reinen Gold der Psychoanalyse mehr Unheil gestiftet als Klarheit geschaffen, dabei drückt sie vermutlich nicht viel mehr aus als die hohe Wertschätzung, die Freud seiner eigenen Erfindung entgegengebracht hat. Wir mögen sie ihm von Herzen gönnen und teilen sie ja auch mit ihm. Allenfalls kommt auch noch sein Dilemma dazu: die Psychoanalyse zwar nicht zu verwässern, aber doch ihre Reichweite über das ursprüngliche Ghetto (Ghetto im doppelten Sinne) hinaus zu steigern, ein Dilemma, das die Psychoanalyse auch nach seinem Tod nicht zu lösen vermochte. Das Reinheitsgebot, als welches diese Metapher (miss)verstanden wurde, hat zu Absurditäten geführt, wie der, dass der Analytiker am besten gar nichts sage und so auch nichts falsch machen könne. 
Gründlicher als dieses Selbst(miss)verständnis kann sie auch der grösste Feind der Psychoanalyse nicht zur Schnecke machen. Auch wenn man die gesellschaftlichen Voraussetzungen dieser Idee, unter denen sie entstanden ist, nachzeichnen kann, bleibt eine nachhaltige Verwunderung darüber zurück, wie die internationale Psychoanalyse jahrzehntelang glauben konnte, mit dieser sterilen Haltung dem Wildwuchs des Unbewussten und Triebhaften gewachsen zu sein. Dies kam man beim besten Willen nur als Gegenbesetzung verstehen - oder bildlicher gesprochen, als Schutzschild der Analytiker gegenüber einer Bedrohung, der sich auszusetzen sie sich weigern, weil sie um ihre Seelenruhe fürchten.

Wie Passett weist auch Weissberg nachdrücklich darauf hin, dass es darum geht, dem Unbewussten zur Sprache zu verhelfen und auf es zu hören - unter welchen äusseren Umständen auch immer. Wie der Geist bekanntlich weht, von wannen er will, so manifestiert sich auch das Unbewusste dort, wo es ihm passt. Die beiden klinischen Vignetten Passetts dokumentieren das eindrücklich. Das psychoanalytische Setting ist zwar von seinem Erfinder ausdrücklich daraufhin angelegt, den Auftritt des Unbewussten zu provozieren, aber dieses erlaubt sich gelegentlich auch einen fulminanten Auftritt auf der Bühne der Psychotherapie, dass die reinen Analytiker grün vor Neid werden könnten. Von der - von Passett geschätzten - genetischen Identität zwischen Analyse und Therapie entfällt vermutlich tatsächlich der Hauptteil auf die schlichte Tatsache, dass beides Tummelplätze des Unbewussten sind.

Ich denke, die Vorträge haben deutlich aufgezeigt, wie sehr die Unterschiede zwischen Psychoanalyse und Psychotherapie durch divergierende Interessen und unreflektierte Ideologien zu einem künstlichen Gegensatz hochstilisiert worden sind. Weder trägt ausschliesslich die Deutung zur Wirkung der Liegekur bei, noch ist sie frei von den Elementen, welche die Wirksamkeit der Psychotherapie ausmachen. Aus reinem Gold besteht auch sie nicht. Indem sie diese Künstlichkeit ins Bewusstsein riefen, leisten die Zyklusvorträge einen Beitrag, eine Debatte zu deblockieren, die das PSZ zu lähmen droht. Sie schaffen Raum dafür, dass die Diskussion um die Zukunft des PSZ endlich in Angriff genommen werden kann. Für ein PSZ, das (ob es ihm gefällt oder nicht) realisiert, dass sich die Pionierphase seiner Existenz dem Ende zuneigt, und das sich souverän als ein psychoanalytisches Institut neben anderen positioniert.

Ich sehe die Zukunft des PSZ als eine Brutstätte des Unbewussten und als eine Ansammlung von leidenschaftlichen, mitfühlenden, neugierigen und unerschrockenen Leuten, die mit Spannung und einigem Wissen auf seine Manifestationen hören und sie zum Wohl ihrer Analysanden entschlüsseln. Sie wollen keine büro- 
kratischen Verwalter der seelischen Gesundheit werden, auch wenn sie dem Appell Freuds aus dem fernen 1918 Folge leisten. Als Leidenschaftliche werden sie ihren Platz behaupten, auch und gerade neben all jenen Institutionen, die vorgeben, genau zu wissen, wie viel Stunden das Unbewusste pro Woche benötigt, um sich aus den Abgründen der Seele hervorlocken zu lassen, und wie lange eine Analyse braucht, um überhaupt eine solche genannt werden zu dürfen. 